ORIGINAL ARTICLE

\title{
Perceived muscular tension, job strain, physical exposure, and associations with neck pain among VDU users; a prospective cohort study
}

\author{
J Wahlström, M Hagberg, A Toomingas, E Wigaeus Tornqvist
}

Occup Environ Med 2004;61:523-528. doi: 10.1136/oem.2003.009563

See end of article for authors' affiliations

Correspondence to: Dr J Wahlström, Occupational and Environmental Medicine, Box 414, SE-405 30 Göteborg, Sweden; jens.wahlstrom@ymk.gu.se

Accepted 8 October 2003

\begin{abstract}
Aims: To determine whether perceived muscular tension, job strain, or physical exposure are associated with increased risk of developing neck pain among VDU users.

Methods: A baseline questionnaire was answered by 1283 respondents, of whom 671 were free from neck pain at baseline. Perceived muscular tension, job strain, and physical exposure were assessed at baseline. Information about newly developed neck pain was collected in 10 follow up questionnaires and the case definition was the first report of such pain in any of the follow up questionnaires. Median follow up time was 10.9 months.

Results: Both men and women who perceived muscular tension at least a few times per week, compared to those who had not perceived muscular tension the preceding month, had an incidence rate ratio (IRR) of 1.9 (95\% Cl 1.25 to 2.93) for developing neck pain, when stratifying for sex. High perceived muscular tension was associated with an increased risk (IRR 1.6, $95 \% \mathrm{Cl} 1.02$ to 1.91), even when controlling for job strain, physical exposure, and age in the model stratified by sex.

Conclusions: Perceived muscular tension was associated with an increased risk of developing neck pain among VDU users. The combination of high job strain and high perceived muscular tension was associated with higher risk of developing neck pain than the combination of high physical exposure and high perceived muscular tension. There was an indication of an excess risk due to interaction between high physical exposure and high job strain.
\end{abstract}

$\mathrm{N}$ eck pain is believed to have a multifactorial aetiology, with physical, psychosocial and individual factors interacting in the development of these disorders, ${ }^{1-7}$ though some investigators conclude that psychosocial factors are of greater importance than physical. ${ }^{8} 9$ Several theoretical models of how these factors relate to each other and their associations with musculoskeletal pain have been proposed..$^{10-14}$ There are no generally accepted criteria for classification of pain in the neck/shoulder region, but the umbrella term cervicobrachial disorders has been used. ${ }^{15}$

In previous cross-sectional studies, we found that both physical and psychosocial factors were associated with muscle activity, measured with electromyography (EMG). ${ }^{16-19}$ We have also found positive associations between perceived muscular tension and muscle activity assessed with EMG. ${ }^{18}$ Theorell and colleagues ${ }^{20}$ reported that perceived muscular tension was associated with symptoms from the back, neck, and shoulders in a cross-sectional study. Crosssectional studies of populations characterised by low biomechanical exposure-that is, customer service work, have observed associations between perceived general tension and musculoskeletal symptoms in the neck/shoulder region..$^{21} 22$

Whether perceived muscular tension is a risk factor or an intermediate in the development of musculoskeletal symptoms is unknown, though Holte and colleagues ${ }^{23}$ suggested perceived general tension to be an intermediate response to organisational and psychosocial factors. In the present study perceived muscular tension has been treated as a risk factor.

The aim of the present study was to determine whether perceived muscular tension, job strain, physical exposure, or individual factors, or combinations of these factors, were associated with increased risk of developing neck pain among VDU users.

\section{SUBJECTS AND METHODS}

\section{Study group}

Information about symptoms, work organisation, work content, and physical, psychosocial, and individual factors during the preceding month was collected by questionnaire at baseline. Information about newly developed musculoskeletal symptoms from the neck/scapular region (fig l) was collected in 10 follow up questionnaires. With support from the employers and together with the occupational health care centres of 46 different worksites, work groups or departments were invited to participate in the study. The worksites differed in size, the smallest including only seven persons and the largest 260. None of the worksites that were invited refused to participate. The study group represented both the private and the public sector. A list of employees at the worksite was established, so that employees on short term leave were also included. The questionnaire was distributed to all employees at the different worksites by ergonomists at the occupational health care centres. The ergonomists were also responsible for checking that the questionnaires were properly filled in, and for collecting them. The study was approved by the local ethics committee at the Karolinska Institute and the regional ethics committee at Göteborg University.

The study base consisted of 1529 computer users (634 men and 895 women). The questionnaire was answered by 1283 subjects (498 men (79\%) and 785 women $(88 \%)$ ). Of these, $671(52 \%)$ were free from neck pain at baseline, with an even distribution between men (51\%) and women (49\%) (fig 2). Among those free from neck pain at baseline, the mean age for the men was 43 years (range 20-65) and for the women was 45 years (range 22-65). All major occupational groups in the Swedish total workforce that spent half or more of their day using a computer ${ }^{24}$ were represented in our study group. 


\section{Main messages}

- Perceived muscular tension was associated with an increased risk for developing neck pain among VDU users.

- For neck pain a dose-response relation with perceived muscular tension was observed.

The major female occupational categories were management/ administration (48\%), graphic design (9\%), library staff (9\%), medical secretary $(7 \%)$, occupational health staff $(7 \%)$, and academic faculty $(7 \%)$. The major male categories were management/administration (31\%), engineering (29\%), academic faculty $(10 \%)$, graphic design $(9 \%)$, service staff $(9 \%)$, and computer support $(6 \%)$. The men had a slightly higher body mass index than the women, 24.8 and 23.4, respectively. The women reported the amount of VDU work in relation to the total work time to be $47.6 \%$, compared to $41.8 \%$ for the men.

\section{Questionnaire}

At baseline the occurrence of musculoskeletal symptoms and occupational exposures during the preceding month and individual factors were assessed by means of a printed questionnaire. The collected information included working hours, work content (variation of work tasks, hours/week of computer work, work with a non-keyboard input device, and data/text entry), physical exposures (amount of precision work and repetitive work), and psychosocial exposures (job demands and decision latitude). Individual factors such as civil status, age, educational level, and lifestyle factors were also included. Information about neck pain was collected by means of the questionnaire at baseline and in the follow up questionnaires. Incidence data were assessed with 10 monthly follow up questionnaires regarding pain in the neck/scapular region. The questions referred to the time period since the preceding questionnaire. The time period usually covered approximately one month, but for some respondents this time period could be longer due to vacations, business journeys, or other reasons for absence. If a follow up questionnaire was not answered before the next one appeared, the preceding questionnaire was omitted and that follow up occasion considered missing. However, the time frame considered for reporting pain covered the period since the last questionnaire was answered.

A question regarding muscular tension was worded as follows: "Have you, during the past month, experienced muscular tension (for example: wrinkled your forehead, ground your teeth, raised your shoulders)?". The response scale comprised four categories: never, a few times, a few times per week, one or several times per day. Respondents were classified into three groups: high tension (a few times per week or one or several times per day), medium tension (a few times), or low tension (never), which were used in the statistical analysis.

Physical exposure was assessed at baseline with two questions: (1) During the last month, have you carried out precision work (for example, work with precision tools, computer mouse, or the like) for altogether more than half an hour per day? (2) During the last month, have you carried out work tasks where the same hand or finger movements were repeated several times a minute (for example, typing, keyboard work, sorting paper) for altogether more than half an hour per day)? The response scales comprised four categories: never or almost never, a few days per month, a few days per week, daily or almost daily. Subjects who

\section{Policy implications}

- Intervention studies may be designed to lower the perceived muscular tension and as a result the incidence of neck pain.

- Intervention strategies more focused on the individual may be an alternative in occupations characterised by low force requirements

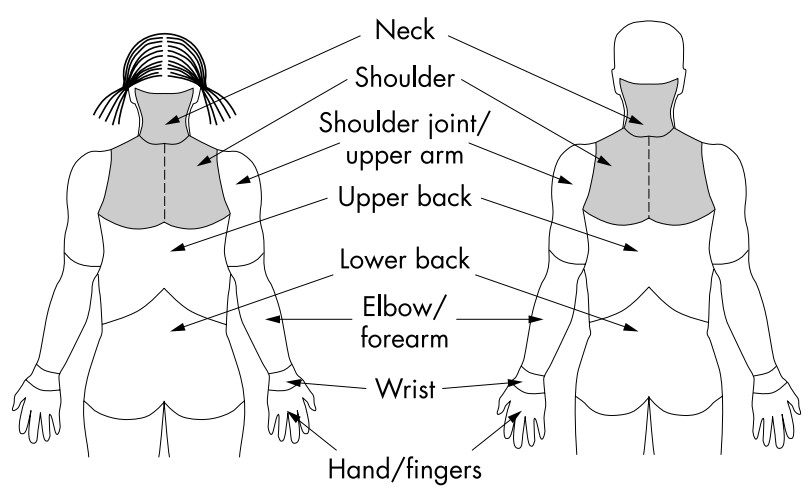

Figure 1 Definition of the different body regions in the symptom recording questionnaires (female and male version). Neck pain was defined as reporting pain or aches in the neck and/or shoulders (shaded area) for three days or more the preceding month.

reported precision and repetitive work daily or almost daily also reported the average time of exposure per day in per cent of the total work time. The median daily exposure was used as the cut-off point for low/high exposure. Hence, subjects with an average time of daily exposure greater than the median value were classified as exposed and the others as unexposed to the two factors, respectively. Respondents were then classified into three groups of physical exposure: high physical exposure (high exposure to both precision and repetitive work), medium physical exposure (high exposure to precision or repetitive work), and low physical exposure (low exposure to both precision and repetitive work).

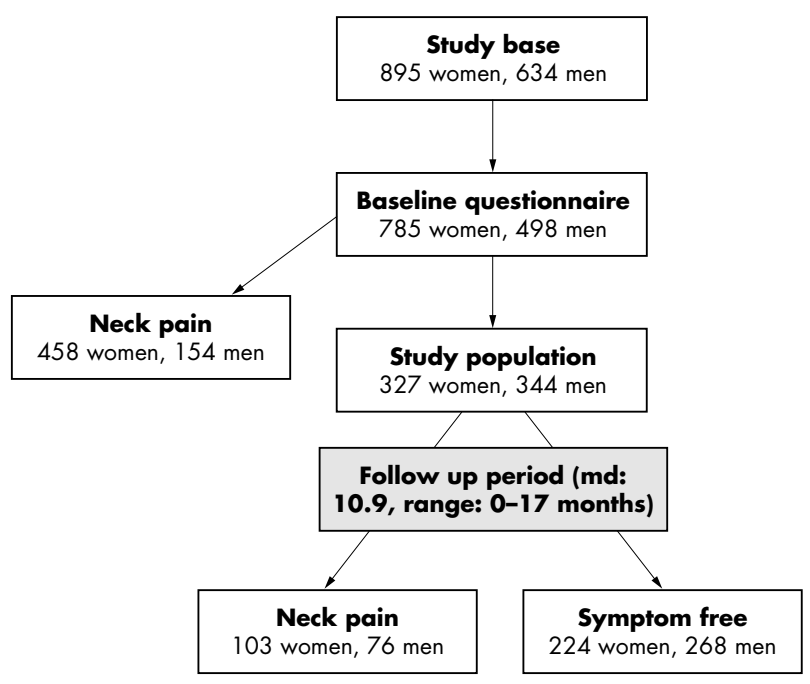

Figure 2 Flow chart of participant eligibility for analyses of incident neck pain. 
To assess the psychosocial exposure, central components from the model suggested by Karasek and Theorell were used. ${ }^{25}$ The model is built from three variables: psychological demands (five items), decision latitude (six items), and social support ( six items). In this study, psychological demands and decision latitude were assessed using a Swedish short version of the Job Content Questionnaire. ${ }^{26}$ For demands and decision latitude, responses were given on a four point scale ranging from "yes, often" to "no, never". The median value of the demand and decision latitude scores, respectively, was used as a cut-off point for low/high exposure. Respondents were then classified into three groups of job strain: high job strain (high demands and low decision latitude), medium strain (high demands and high decision latitude or low demands and low decision latitude), and low strain (low demands and high decision latitude).

Age was trichotomised into a young (18-34), a middle (35$44)$, and an old $(\geqslant 45)$ age group.

\section{Analyses}

Neck pain at baseline was defined as reported pain or aches in the neck and/or scapular area (fig l) for three days or more during the preceding month. The case definition was the first report of such pain in any of the 10 follow up questionnaires. Cases only contributed person-time to the analyses until they were classified as cases.

All statistical analyses were performed separately for men and women or stratified by sex. Incidence rate ratios (IRR (hazard ratio)) with 95\% confidence intervals (95\% CI) were computed using Cox proportional hazard models (proc phreg, SAS v. 8.2). Kaplan-Meier survival curves were obtained by means of the statistical software JMP version 4.0.4. The potential excess risk attributable to interaction was assessed between the exposures "perceived muscular tension" and "job strain", "perceived muscular tension" and "physical exposure", and "job strain" and "physical exposure" by measuring departure from additivity of effect by the method proposed by Rothman and Greenland. ${ }^{27}$

\section{RESULTS}

The median follow up time was 10.9 months (range 0-17.5); 179 subjects ( 103 women and 76 men) developed neck pain during follow up (table 1). The incidence rate of the first episode of neck pain was 36 new cases per 100 person-years.
Both men and women who perceived muscular tension at least a few times per week compared to those who had not perceived muscular tension the preceding month had an IRR of 1.9 (95\% CI 1.25 to 2.93) for developing neck pain, when stratifying for sex (table 2). Respondents with high job strain had an IRR of 1.6 (95\% CI 1.03 to 2.61) for developing neck pain compared to those with low job strain when stratifying for sex (table 2).

High perceived muscular tension was associated with an increased risk (IRR 1.6, 95\% CI 1.02 to 2.48), even when controlling for job strain, physical exposure, and age in the model stratified by sex (table 2).

When combining different risk factors, an IRR of 4.0 (95\% CI 1.6 to 10.0) was observed for respondents with high perceived muscular tension and high job strain compared to those with low perceived muscular tension and low job strain (table 3, fig 3). Combining high job strain and high physical exposure resulted in an IRR of 2.7 (95\% CI 1.2 to 5.9). However, for the combination of job strain and physical exposure, an excess risk attributable to the interaction of 0.75 was indicated (table 3 ).

\section{DISCUSSION}

Our a priori hypothesis was that individuals who perceived muscular tension at least a few times per week had an increased risk of developing neck pain. The results from both the unadjusted and adjusted analyses supported this hypothesis. This is the first report where perceived muscular tension has been observed as an independent risk factor or intermediate for development of neck pain in a prospective study. Thus, intervention studies may be designed to lower the perceived muscular tension and as a result lower the incidence of neck pain. There may be a need for more interventions in the workplaces more specifically addressed to the individual than to the workplace, since the perception of muscular tension probably arises from both workplace factors and individual factors. This issue has also been addressed by Holte and Westgaard, ${ }^{93}$ who pointed out that there may be a need to assess factors which focus more on the individual translation of the exposure into an individual response than the more traditional risk factors like job strain and physical exposure. New intervention strategies, focused more on the individual, may be an alternative in occupations characterised by work that entails low force requirements but

\begin{tabular}{|c|c|c|c|c|}
\hline \multirow[b]{3}{*}{ Risk factor } & \multicolumn{4}{|c|}{ Neck pain during follow up } \\
\hline & \multicolumn{2}{|l|}{ Men $(n=344)$} & \multicolumn{2}{|c|}{ Women $(n=327)$} \\
\hline & No $(n=268)$ & Yes $(n=76)$ & No $(n=224)$ & Yes $(n=103)$ \\
\hline \multicolumn{5}{|l|}{ Muscular tension } \\
\hline Low & 95 & 20 & 61 & 16 \\
\hline Medium & 117 & 29 & 106 & 54 \\
\hline High & 51 & 23 & 55 & 32 \\
\hline \multicolumn{5}{|l|}{ Job strain } \\
\hline Low & 100 & 20 & 44 & 15 \\
\hline Medium & 122 & 42 & 117 & 54 \\
\hline High & 39 & 12 & 53 & 32 \\
\hline \multicolumn{5}{|l|}{ Physical exposure } \\
\hline Low & 169 & 45 & 103 & 35 \\
\hline Medium & 65 & 20 & 63 & 40 \\
\hline High & 28 & 11 & 43 & 22 \\
\hline \multicolumn{5}{|l|}{ Age } \\
\hline Young (18-34) & 75 & 23 & 49 & 14 \\
\hline Middle (35-44) & 66 & 26 & 49 & 24 \\
\hline Old $(\geqslant 45)$ & 127 & 27 & 126 & 65 \\
\hline
\end{tabular}




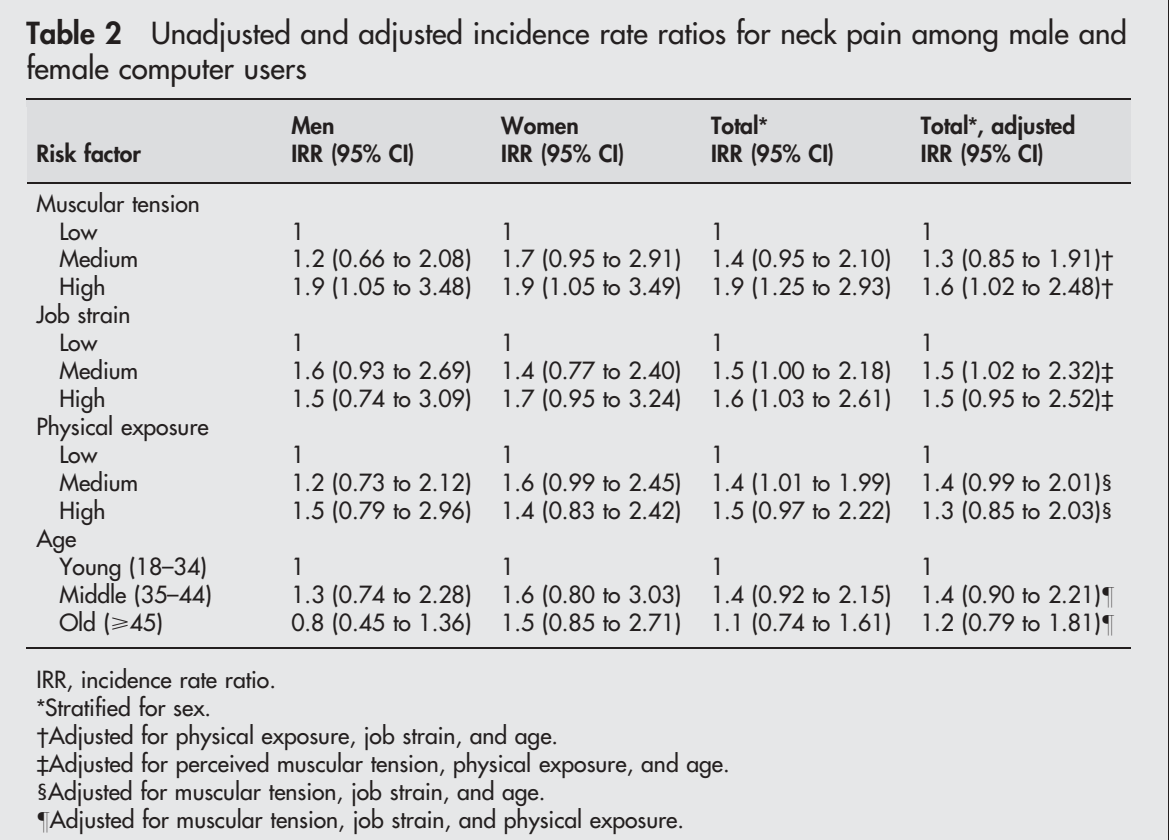

where the physical exposure could still be high regarding precision and repetitive demands, for example, VDU work.

In the present study, an IRR of 1.6 was observed for those with high perceived muscular tension when controlling for other factors. If the work environment is characterised by high job strain and the risk of developing neck pain is greater among individuals who react with muscular tension compared to those who do not, perceived muscular tension would be considered an intermediate or an early sign of neck pain. The results from the present study supports the ecological model proposed by Sauter and Swanson. ${ }^{14}$ The model hypothesises that psychological strain produces muscle tension and moderates the relation between biomechanical strain and musculoskeletal symptoms. Perceived muscular tension could be an early sign in the phase of detection of symptoms, but it is likely that perceived muscular tension could also arise from psychosocial and individual factors, which is not hypothesised in the model from Sauter and Swanson. If perceived muscular tension is an intermediate or an early sign of neck pain, it could be used as part of a survey method to identify work environments with high risks, so that interventions could be made before individuals develop

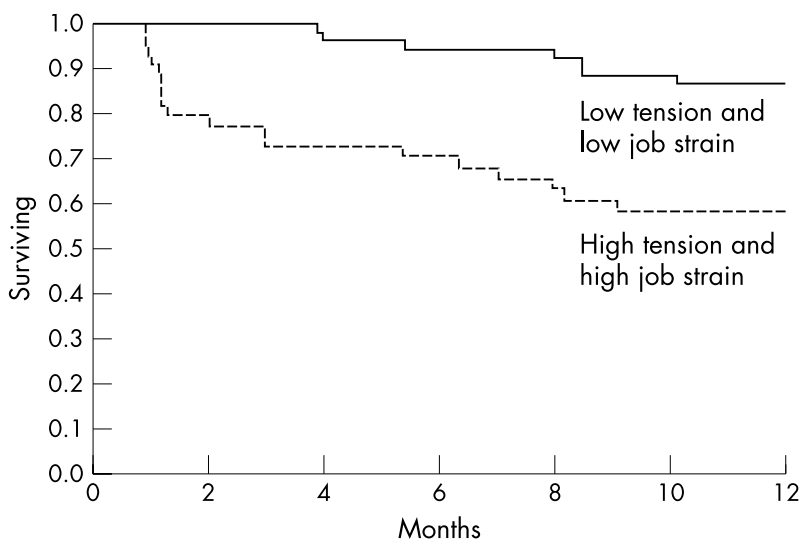

Figure 3 Kaplan-Meier survival curve for men and women (unadjusted and unstratified) with high perceived muscular tension and high job strain compared to those with low perceived muscular tension and low job strain. pain. However, to elucidate whether perceived muscular tension is a risk factor or an intermediate for neck pain, prospective studies are necessary to clarify whether job strain is followed by perceived muscular tension and if there are other factors influencing the perception of muscular tension.

The combination of high perceived muscular tension and high job strain resulted in a higher risk estimate compared to the combination of perceived muscular tension and physical exposure, IRR 4.0 and 1.9 respectively. The items used for describing the physical exposure were amount of precision and repetitive work. The way the different questions were asked and the construction of the variable physical exposure implies that it could be associated with hours/day of VDU work. Lack of association between musculoskeletal symptoms in the neck and daily hours of VDU work has previously been reported. ${ }^{28}$ However, there are other dimensions of the physical working environment than the hours/day of VDU work that are considered to be risk factors for neck pain; for example, absence of arm support, height of the keyboard in relation to elbow height, height of the monitor, and lighting conditions. ${ }^{28} 29$

A possible interaction was found for coexposure between high job strain and high physical exposure, indicated by an excess risk attributable to interaction of 0.75 . Only a few studies have investigated possible interaction effects between physical and psychosocial exposure and increased risk for musculoskeletal symptoms. However, Devereux and coworkers $^{30}$ observed a potential interaction between high psychosocial and high physical exposure in a cross-sectional study of both blue-collar and white-collar workers which further increased the risk of musculoskeletal symptoms in the upper extremities. Wigaeus Tornqvist and colleagues ${ }^{31}$ reported an indication of an excess risk due to interaction between job strain and VDU work for seeking care because of neck or shoulder disorders.

In the current study, $48 \%$ reported prevalent neck pain (that is, during the past month). Other studies of VDU users have reported both higher ${ }^{32}$ and lower ${ }^{33}{ }^{34}$ prevalences of neck pain/musculoskeletal symptoms. The differences between studies could be due to differences in populations studied, the time periods used in the period prevalence calculations, or in the criteria used for defining pain or symptoms. 
Table 3 Combinations of different risk factors for neck pain among male and female computer users, stratified for sex and adjusted for age

\begin{tabular}{|c|c|c|c|}
\hline \multirow[b]{2}{*}{ Risk factor } & \multicolumn{3}{|l|}{ Men and women } \\
\hline & HR $(95 \% \mathrm{Cl})$ & Events/censored & Excess risk \\
\hline \multicolumn{4}{|c|}{ Perceived muscular tension and job strain* } \\
\hline Low tension, low strain & 1 & $7 / 56$ & \\
\hline High tension, low strain & $3.3(1.1$ to 9.5$)$ & $8 / 18$ & \\
\hline Low tension, high strain & $2.5(0.90$ to 6.8$)$ & $9 / 23$ & \\
\hline High tension, high strain & $4.0(1.6$ to 10.0$)$ & $19 / 28$ & -0.19 \\
\hline \multicolumn{4}{|c|}{ Perceived muscular tension and physical exposure } \\
\hline Low tension, low physical & 1 & $20 / 90$ & \\
\hline High tension, low physical & $1.7(0.92$ to 3.3$)$ & $23 / 52$ & \\
\hline Low tension, high physical & $0.83(0.27$ to 2.6$)$ & $4 / 17$ & \\
\hline High tension, high physical & $1.9(0.86$ to 4.2$)$ & $12 / 18$ & 0.17 \\
\hline \multicolumn{4}{|l|}{ Job strain and physical exposuref } \\
\hline Low strain, low physical & 1 & $17 / 89$ & \\
\hline High strain, low physical & $1.1(0.51$ to 2.5$)$ & $14 / 49$ & \\
\hline Low strain, high physical & $0.54(0.12$ to 2.4$)$ & $2 / 15$ & \\
\hline High strain, high physical & $2.7(1.2$ to 5.9$)$ & $15 / 17$ & 0.75 \\
\hline \multicolumn{4}{|c|}{$\begin{array}{l}\text { Note that only respondents with low exposure for both risk factors and high exposure for one or both risk factor } \\
\text { were included, all other possible exposure combinations being excluded. } \\
\text { HR, hazard ratio. } \\
\text { *Adiusted for physical exposure and age. } \\
\text { †Adjusted for job strain and age. } \\
\text { †Adjusted for perceived muscular tension and age. }\end{array}$} \\
\hline
\end{tabular}

In an earlier paper ${ }^{35}$ investigating cross-sectional associations between exposure and musculoskeletal symptoms from the same study base $(\mathrm{n}=1283)$ as used in the present paper, it was observed that women experienced a higher prevalence of musculoskeletal symptoms than men in all body regions. It has been pointed out that it is important to distinguish between crude risk differences between the sexes and the effects of occupational exposures on musculoskeletal symptoms, and to perform separate analyses for men and women. $^{35}$ In the present study, there were no major differences in risk estimates for developing neck pain between men and women in any of the studied exposure variables.

\section{Limitations}

The response rate in the baseline questionnaire was $84 \%$ and must be considered rather good. However, $31 \%$ of the men and $58 \%$ of the women who answered the baseline questionnaire reported neck pain, and were not included in the present study population. This could have biased the results towards an underestimation of the incidence rates (that is, healthy worker effect), since the participants in the study could have been less prone to develop neck pain than those who had neck pain at baseline. In addition to this, individuals who were already on long term sick-leave at baseline were not included in the study base. Women had almost a twofold prevalence of neck pain compared to men at baseline and this could lead to an underestimation of incidence rates among the women compared to the men, as well as an underestimation of the risk estimates among the women. It is thus important to distinguish between the sexes in future studies. Another limitation was the inclusion of individuals with prior experience of VDU work. However, it will be difficult to solve this problem since exposure to VDU use starts in childhood.

Possible confounding factors not controlled for in the multivariate analyses could be previous history of neck pain $^{534}$ and duration of computer work, ${ }^{36}$ which in previous studies have been observed to be risk factors. If perceived muscular tension is more common among those with a previous history of neck pain the observed risk estimates for perceived muscular tension may be overestimated. The results are based on both self reported exposures at baseline and self reported symptoms during follow up, which could have led to either underestimation or overestimation of the risk estimates. The different exposure variables were assessed at baseline and there might have been a risk that some of the exposure variables as well as perceived muscular tension changed during the follow up period.

\section{Conclusions}

Perceived muscular tension was associated with an increased risk of developing neck pain among VDU users, which implies that intervention studies may be designed to lower the perceived muscular tension and as a result lower the incidence of neck pain. The results also suggested that the combination of high job strain and high perceived muscular tension was associated with a higher risk of developing neck pain than the combination of high physical exposure and high perceived muscular tension. An indication of an excess risk due to interaction between high physical exposure and high job strain was also observed.

\section{ACKNOWLEDGEMENTS}

Financial support was obtained from the Swedish Work Environmental Fund. We are also grateful to Maud Hagman and Catarina Karlberg for assistance with the statistical database and to Professor Roland Kadefors for his valuable comments on the manuscript.

\section{Authors' affiliations}

J Wahlström, M Hagberg, E Wigaeus Tornqvist, Department of Occupational Medicine at the Sahlgrenska Academy at Göteborg University, Sweden

A Toomingas, National Institute for Working Life, Stockholm, Sweden

\section{REFERENCES}

1 Leclerc A, Niedhammer I, Landre MF, et al. One-year predictive factors for various aspects of neck disorders. Spine 1999;24:1455-62.

2 Andersen $\mathrm{JH}$, Kaergaard A, Frost $\mathrm{P}$, et al. Physical, psychosocial, and individual risk factors for neck/shoulder pain with pressure tenderness in the muscles among workers performing monotonous, repetitive work. Spine 2002;27:660-7.

3 Ariens GA, Bongers PM, Douwes $M$, et al. Are neck flexion, neck rotation, and sitting at work risk factors for neck pain? Results of a prospective cohort study. Occup Environ Med 2001:58:200-7.

4 Ariens GA, Bongers PM, Hoogendoorn WE, et al. High quantitative job demands and low coworker support as risk factors for neck pain: results of a prospective cohort study. Spine 2001;26:1896-901. 
5 Fredriksson K, Alfredsson L, Köster M, et al. Risk factors for neck and upper limb disorders: results from 24 years of follow up. Occup Environ Med 1999;56:59-66

6 Fredriksson K, Alfredsson L, Bildt Thorbjörnsson C, et al. Risk factors for neck and shoulder disorders: a nested case-control study covering a 24 -year period. Am J Ind Med 2000;38:516-28.

7 Linton SJ. A review of psychological risk factors in back and neck pain. Spine 2000;25:1148-56.

8 Palmer KT, Walker-Bone K, Griffin MJ, et al. Prevalence and occupational associations of neck pain in the British population. Scand J Work Environ Health $2001 ; 27: 49-56$.

9 Holte KA, Westgaard RH. Further studies of shoulder and neck pain and exposures in customer service work with low biomechanical demands. Ergonomics 2002;45:887-909.

10 Armstrong TJ, Buckle P, Fine $\amalg$, et al. A conceptual model for work-related neck and upper-limb musculoskeletal disorders. Scand J Work Environ Health 1993;19:73-84.

11 Huang GD, Feuerstein M, Sauter SL. Occupational stress and work-related upper extremity disorders: concepts and models. Am J Ind Med 2002;41:298-314.

12 Westgaard RH. Effects of physical and mental stressors on muscle pain Scand J Work Environ Health 1999;25(suppl 4):19-24.

13 Westgaard RH, Winkel J. Guidelines for occupational musculoskeletal load as a basis for intervention: a critical review. Appl Ergon 1996;27(2):79-88.

14 Sauter SL, Swanson NG. An ecological model of musculoskeletal disorders in office work. In: Moon SD, Sauter SL, eds. Beyond biomechanics: psychosocial aspects of musculoskeletal disorders in office work. London: Taylor \& Francis, 1996:3-21

15 Hagberg M, Silverstein B, Wells R, et al. Work related musculoskeletal disorders (WMSDs): a reference book for prevention. London: Taylor \& Francis, 1995.

16 Wahlström J, Hagberg M, Johnson PW, et al. Influence of time pressure and verbal provocation on physiological and psychological reactions during work with computer mouse. Eur J Appl Physiol 2002;87:257-63.

17 Wahlström J, Svensson J, Hagberg M, et al. Differences between work methods and gender in computer mouse use. Scand J Work Environ Health 2000:26:390-7.

18 Wahlström J, Lindegård A, Ahlborg Jr G, et al. Associations between physica load and psychological demands, emotional stress and perceived muscular tension during VDU work in occupational settings. Int Arch Occup Environ Health 2003;76:584-90.

19 Lindegård A, Wahlström J, Hagberg M, et al. The impact of working technique on physical loads - an exposure profile among newspaper editors. Ergonomics 2003;46:598-615.

20 Theorell T, Harms-Ringdahl K, Ahlberg-Hulten G, et al. Psychosocial job factors and symptoms from the locomotor system - a multicausal analysis. Scand J Rehabil Med 1991;23:165-73.
21 Holte KA, Westgaard RH. Daytime trapezius muscle activity and shoulderneck pain of service workers with work stress and low biomechanical exposure. Am J Ind Med 2002;41:393-405

22 Vasselien O, Holte KA, Westgaard RH. Shoulder and neck complaints in customer relations: individual risk factors and perceived exposures at work. Ergonomics $2001 ; 44: 355-72$.

23 Holte KA, Vasseljen O, Westgaard RH. Exploring perceived tension as a response to psychosocial work stress. Scand J Work Environ Health 2003;29:124-33.

24 Ekman A Andersson A, Hagberg M, et al. Gender differences in musculoskeletal health of computer and mouse users in the Swedish workforce. Occup Med (Lond) 2000;50:608-13.

25 Karasek R, Theorell T. Healthy work. New York: Basic Books, 1990.

26 Theorell T, Michélsen $\mathrm{H}$, Nordemar R. Tre arbetsmiliöindex som använts Stockholmsundersökningen [in Swedish]. In: Hagberg M, Hogstedt C, eds. Stockholmsundersökningen 1. Stockholm: MUSIC Books, 1991:150-4.

27 Rothman KJ, Greenland S. Modern epidemiology, 2nd edn. Philadelphia: Lippincott-Raven, 1998.

28 Marcus M, Gerr F, Monteilh C, et al. A prospective study of computer users: II. Postural risk factors for musculoskeletal symptoms and disorders. Am J Ind Med 2002;41:236-49.

29 Aarås A, Horgen G, Bjørset $\mathrm{HH}$, et al. Musculoskeletal, visual and psychosocial stress in VDU operators before and after multidisciplinary ergonomic interventions. A 6 years prospective study-Part II. Appl Ergon 2001;32:559-71.

30 Devereux JJ, Vlachonikolis IG, Buckle PW. Epidemiological study to investigate potential interaction between physical and psychosocial factors at work that may increase the risk of symptoms of musculoskeletal disorder of the neck and upper limb. Occup Environ Med 2002;59:269-77.

31 Wigaeus Tornqvist E, Kilbom $\AA$, Vingård $E$, et al. The influence on seeking care because of neck and shoulder disorders from work-related exposures. Epidemiology 2001;12:537-45.

32 Bergqvist U, Wolgast E, Nilsson B, et al. Musculoskeletal disorders among visual display terminal workers: individual, ergonomic, and work organizational factors. Ergonomics 1995;38:763-76.

33 Bernard B, Sauter S, Fine L, et al. Job task and psychosocial risk factors for work-related musculoskeletal disorders among newspaper employees. Scand J Work Environ Health 1994;20(6):417-26.

34 Gerr F, Marcus M, Ensor C, et al. A prospective study of computer users: I. Study design and incidence of musculoskeletal symptoms and disorders. Am J Ind Med 2002;41:221-35.

35 Karlqvist L, Wigaeus Tornqvist E, Hagberg M, et al. Self-reported working conditions of VDU operators and associations with musculoskeletal symptoms: a cross-sectional study focussing on gender differences. Int J Ind Ergon 2002;30:277-94.

36 Punnett L, Bergqvist U. Visual display unit work and upper extremity musculoskeletal disorders. Stockholm: National Institute for Working Life, 1997. 